\title{
Trapping and amplification of unguided mode EMIC waves in the radiation belt
}

\section{Geng Wang ( $\nabla$ wanggeng@hit.edu.cn )}

Institute of Space Science and Applied Technology, Harbin Institute of Technology

\section{Zhonglei Gao}

School of Physics and Electronic Sciences, Changsha University of Science and Technology

\section{Mingyu Wu}

Harbin Institute of Technology, Shenzhen

\section{Guoqiang Wang}

Harbin Institute of Technology, Shenzhen

\section{Sudong Xiao}

Institute of Space Science and Applied Technology, Harbin Institute of Technology, Shenzhen

\section{Yuanqiang Chen}

Department of Geophysics and Planetary Sciences, University of Science and Technology of China

\section{Zhengyang Zou}

Institute of Space Science and Applied Technology, Harbin Institute of Technology

\section{Tielong Zhang}

Institute of Space Science and Applied Technology, Harbin Institute of Technology, Shenzhen

\section{Article}

Keywords: electromagnetic ion cyclotron (EMIC), radiation belt

Posted Date: January 21st, 2021

DOl: https://doi.org/10.21203/rs.3.rs-132689/v1

License: (c) (1) This work is licensed under a Creative Commons Attribution 4.0 International License. Read Full License

Version of Record: A version of this preprint was published at Journal of Geophysical Research: Space Physics on August 27th, 2021. See the published version at https://doi.org/10.1029/2021JA029322. 


\title{
Trapping and amplification of unguided 2 mode EMIC waves in the radiation belt
}

\begin{abstract}
Electromagnetic ion cyclotron (EMIC) waves can cause the scattering loss of the relativistic electrons in the radiation belt. They can be classified into the guided mode and the unguided mode, according to waves propagation behavior. The guided mode waves have been widely investigated in the radiation belt, but the observation of the unguided mode waves have not been expected. Based on the observations of Van Allen Probes, we demonstrate for the first time the existence of the intense unguided mode EMIC waves in the radiation belt. Growth rate analyses indicate that the hot protons with energies of a few hundred keV may provide the free energy for wave growth. The reflection interface formed by the spatial locations of local helium cutoff frequencies can be nearly parallel to the equatorial plane when the proton abundance ratio decreases sharply with $L$-shell. This structure combined with hot protons may lead to the trapping and significant amplification of the unguided mode waves. These results may help to understand the nature of EMIC waves and their dynamics in the radiation belt.
\end{abstract}




\section{${ }_{21}$ Introduction}

The earth radiation belt is dynamically influenced by multiple types of waves, one of them is the electromagnetic ion cyclotron (EMIC) wave, which has been widely investigated in recent decades ${ }^{[1-9]}$, and has been considered to have the potential to remove the relativistic electrons from the radiation belt over a time-scale of several hours ${ }^{[10-20]}$.

The typical EMIC waves have often been observed just below the hydrogen $\left(\mathrm{H}^{+}\right)$gyrofrequencies $\left(f_{\mathrm{cH}+}\right)$, with left-hand polarizations (L-mode) and quasiparallel wave vectors $[3,5,7]$. In the radiation belt, the existence of minor ions $\left(\mathrm{He}^{+}\right.$and $\left.\mathrm{O}^{+}\right)$sperate the cold plasma dispersion curves of EMIC waves into several branches, and the L-mode EMIC waves can belong to two types of propagation modes: one is the guided mode and the other is the unguided mode (Supplementary Fig.1), according to whether the wave group velocity is guided by the ambient magnetic field ${ }^{[21,22]}$. The guided mode waves can propagate in the same $L$-shell within which the substorm injected hot ions are trapped. The anisotropic hot $\mathrm{H}^{+}$with the energy of tens of $\mathrm{keV}$ can resonate with the waves of guided mode, and lead to the wave growth [21, 23-30]. Moreover, the anisotropic $\mathrm{H}^{+}$distributions induced by the magnetospheric compression may also lead to the generation of guided mode EMIC waves close to $f_{\mathrm{cH}+}{ }^{[2,31-35]}$. Therefore, the commonly observed EMIC waves are widely investigated based on the guided mode ${ }^{[36-40]}$.

Simulations have suggested that the convective amplification of the unguided mode waves will not be significant, as the wave normal angle increases very quickly and the waves rapidly move to larger $L$-shell $[21,22,40]$. Moreover, as the wavenumber of the unguided mode is relatively larger than that of the guided mode (Supplementary Fig.1), the resonant energy may exceed the peak energy of the substorm injected hot $\mathrm{H}^{+}$, which does not favor the wave generation.

Up to now, the investigations of unguided mode EMIC waves in the radiation belt have been ignored. However, the hot $\mathrm{H}^{+}$with hundreds of $\mathrm{keV}$ can inject into the ring current during storms ${ }^{[41]}$, theoretically providing the energy source for the generation of unguided mode waves. Moreover, the smaller group velocities of unguided mode waves may lead to the larger convective growth rates, which can favor the wave generation ${ }^{[42]}$.

The motivation of the present study is based on the following questions: Can we find the distinguishable unguided mode EMIC waves that are locally generated below $f_{\mathrm{cH}+}$ ? If so, what's the difference of the generation mechanism between the guided and unguided mode waves? Here we identify the existence of the intense unguided mode EMIC waves in the earth's radiation belt by presenting Van Allen Probe ${ }^{[43]}$ observations. We will propose that the generation of these waves are associated with a new type of wave trapping mechanism, which is controlled by the spatial variation of ion abundance ratios. In the discussion section, we will focus on the question that whether the unguided mode EMIC waves are common in the radiation belt. 


\section{Results}

\section{Overview of the Event}

As shown in Figure 1a, the event was observed by Probe A on 15 December 2015 , during the recovery phase of a magnetic storm, after at least three successive prominent substorms where $A E>500 \mathrm{nT}$. In Figure $1 \mathrm{~b}-\mathrm{c}$, the hot $\mathrm{H}^{+}$ fluxes detected by the Radiation Belt Storm Probes Ion Composition Experiment (RBSPICE) instrument ${ }^{[44]}$ and the Helium, Oxygen, Proton, and Electron (HOPE) mass spectrometer ${ }^{[45]}$, enhance in four distinct energy ranges: below $10 \mathrm{keV}, 10-30 \mathrm{keV}, 30-100 \mathrm{keV}$ and $100-600 \mathrm{keV}$. The peak energy of the hot $\mathrm{H}^{+}$for each energy range decreases with $L$-shell, indicating the energydispersive substorm injected ions ${ }^{[41]}$. From Figure 1d, the satellite passed through the plasmapause at $L \sim 2.87$, and the background electron number density $n_{e}$ which was measured by the High Frequency Receiver (HFR) ${ }^{[46]}$ of the Electric and Magnetic Field Instrument and Integrated Science (EMFISIS) suite ${ }^{[47]}$, dropped from $\sim 100 \mathrm{~cm}^{-3}$ at just outside the plasmapause to $\sim 20$ $\mathrm{cm}^{-3}$ at $L \sim 3.7$. Measured by the triaxial fluxgate magnetometer (MAG) of EMFISIS, the conspicuous $\mathrm{H}^{+}$band waves appear from $L \sim 3.1$ to $L \sim 3.7$, as exhibited in Figure 1e. These waves with quasi-constant frequencies appear to be intense just outside the plasmapause and decay gradually with the increasing $L$-shell.

\section{Wave analyses and Ion Abundance Ratios}

The detailed wave properties are exhibited in Figure 2. In general, the time series of the wave spectra can be divided into three distinct regions based on the observational characteristics, as marked by the blue boxes. The Region A waves appear at MLAT $\sim-1.5$, have the strongest magnetic spectral density $\mathrm{PSD}_{B}$, associated with the quasi-parallel wave normal angle $\psi$ and small compressional ratio $\mathrm{PSD}_{B \|} / \mathrm{PSD}_{B}$, indicating that they were propagating roughly along the axis perpendicular to the equatorial plane. Furthermore, the general direction (shown by the diamonds, which are the spectrum-weighted averages) of the Poynting fluxes appears to be northward for the inner waves, and be southward for the outer waves, as shown in Figure 2f. The Poynting fluxes for almost all these intense waves possess an inward orientation according to Figure 2e. The results of the ellipticity $\epsilon_{B}$ show predominately left-hand polarization, indicating that the waves belong to the L-mode. As the satellite moved away from the Earth, Region B waves were observed in successive time series, but these waves show a decrease in $\mathrm{PSD}_{B}$ of approximately one order. The most outstanding phenomena are recorded in the $\epsilon_{B}$ spectra, where the waves exhibit left-hand polarization inside $L=3.46$ but right-hand polarization outside. There is a sharp boundary that roughly shifts from a higher frequency at inner $L$ to a lower frequency at outer $L$. This phenomenon may indicate the pass-through of the $\mathrm{He}^{+}$crossover frequency $f_{\mathrm{crHe}+}$, and the waves with right-hand polarization should belong to the R-mode. Independent of the polarization, the waves with 

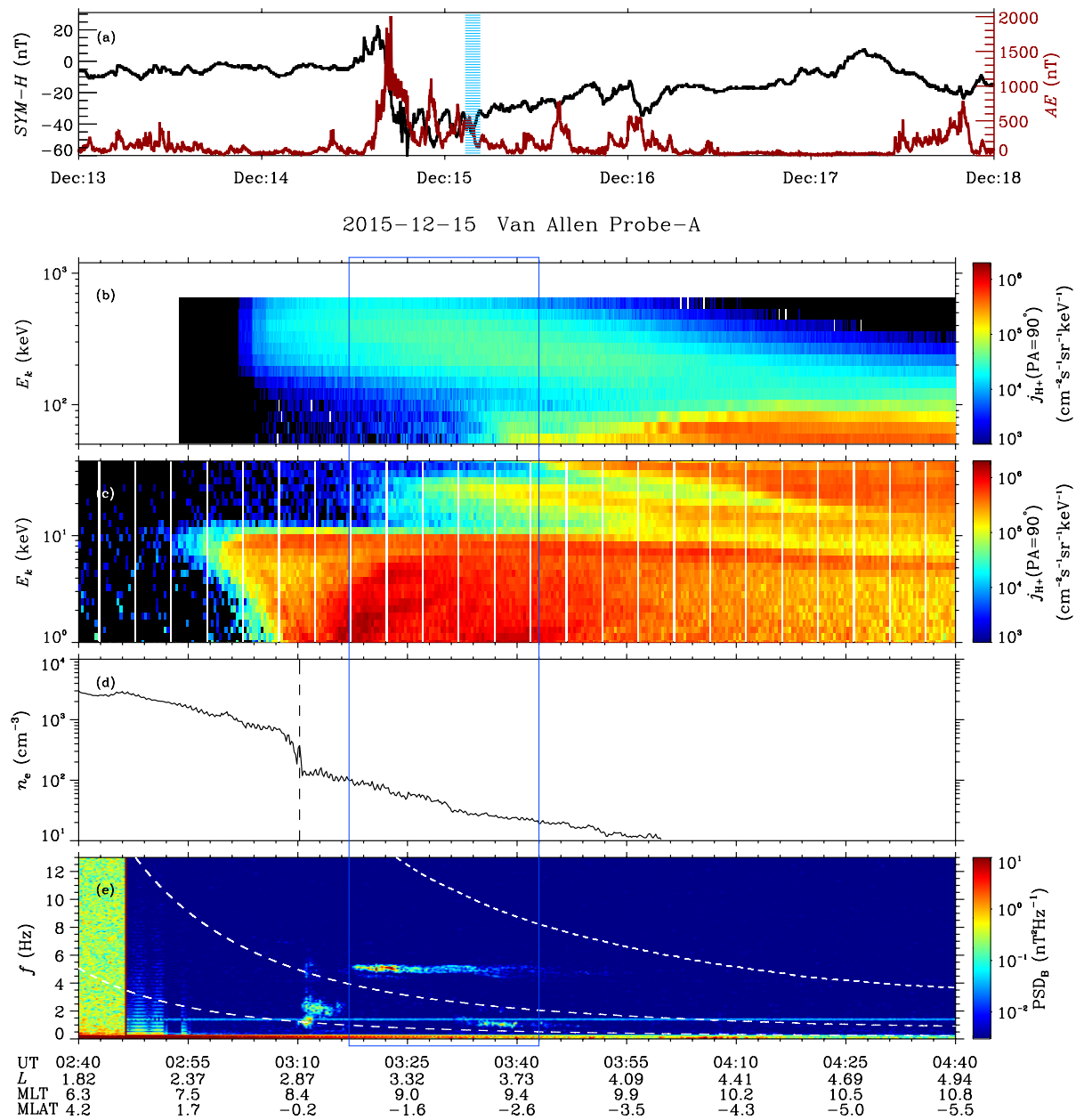

Figure 1: Overview of the background conditions of the wave event recorded by Van Allen Probe A on 15 December 2015 : (a) SYM-H index and AE index (shaded region marks the period of the wave event). (b) Background electron number density $n_{e}$ (dashed lines for the location of the plasmapause). (c,d) Energy-dependent $\mathrm{H}^{+}$differential fluxes $j_{\mathrm{H}+}$ at a $90^{\circ}$ pitch angle measured by RBSPICE and ECT HOPE. (e) Magnetic power spectral density $\mathrm{PSD}_{B}$. The white dashed curves trace the local gyrofrequencies of hydrogen, helium, and oxygen. The blue box marks the period of the wave event. 


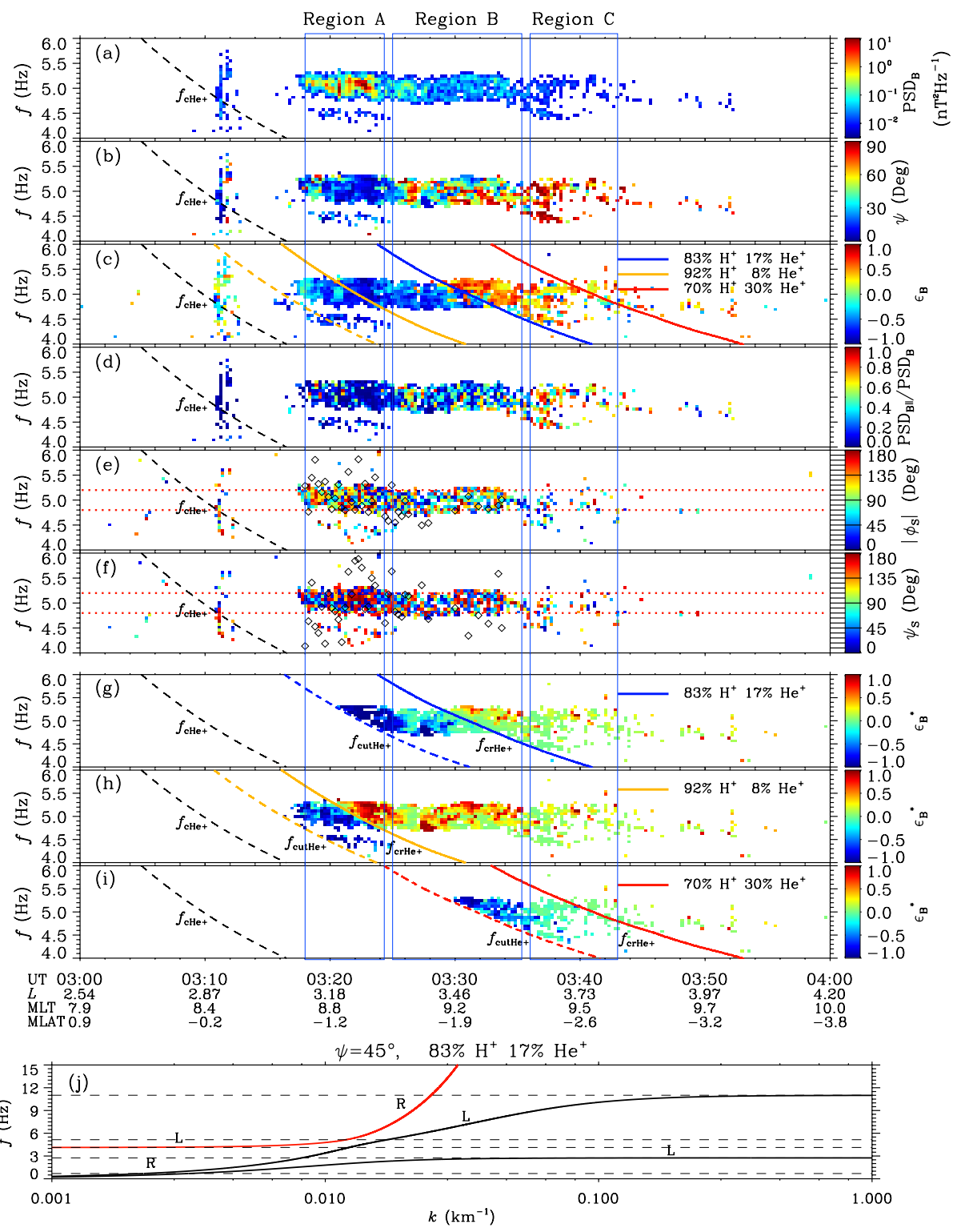

Figure 2: Analyses of wave properties and wave mode. (a) Magnetic power spectral density $\mathrm{PSD}_{B}$. The blue boxes roughly separate the three distinct regions with different observational characteristics. (b) Wave normal angle $\psi$ (unifying the two field-aligned orientations). (c) Wave ellipticity $\epsilon_{B}$. (d) Wave magnetic compression ratio $\mathrm{PSD}_{B \|} / \mathrm{PSD}_{B}$. (e) The azimuthal angle of the Poynting flux $\phi_{S}\left(0^{\circ}\right.$ represents away from the Earth). (f) The angle of the Poynting flux with respect to the ambient magnetic field $\psi_{S}$. Black diamonds in (e) and (f) are the spectrum-weighted averaged angles within the frequency range of $4.8-5.2 \mathrm{~Hz}$ (red dotted lines), and they share the same axis with the corresponding color bars. (g-i) The theretically calculated distribution of the ellipticity $\epsilon_{B}^{*}$ for the given ion abundance ratios. The colored solid and dashed lines trace the local crossover frequencies $f_{\text {crHe+ }}$ and cutoff frequencies $f_{\text {cutHe+ }}$. (j) Dispersion relations under the observed plasma conditions. The red curve represents the observed wave mode. The letter ' $L$ ' and ' $R$ ' denote the left-hand and right-hand polarizations, respectively. 
different values of the wave normal angle $\psi$ or compressional ratio $\mathrm{PSD}_{B \|} /$ $\mathrm{PSD}_{B}$ are highly mixed. Moreover, the spectrum-weighted averaged angles of Poynting fluxes point mainly away from the Earth or along the azimuthal direction (Figure 2e), with a relatively small angle with respect to the equatorial plane (Figure 2f). The outermost waves are presented in Region C, with a wave power 2 orders of magnitude smaller than that of the waves in Region A. The wave vectors are almost perpendicular to the ambient field, the compressional ratios are relatively large, and the polarizations are predominantly right-handed, indicating these are the oblique R-mode waves.

With increasing $L$-shell value, the relative frequency $f / f_{\mathrm{cH}}$ increases for the observed $\mathrm{H}^{+}$band waves with a quasi-constant frequency, and the polarization mode thus changes from L-mode to R-mode. Such a phenomenon indicates that the waves belong to the unguided mode ${ }^{[21]}$, as illustrated in Figure $2 \mathrm{j}$. Here, we perform the plasma diagnostics to verify the wave mode and estimate the ion abundance ratios $\eta_{s}$. As shown by Figure 2c, a series of $f_{\text {crHe+ }}$ (and also cutoff frequencies $f_{\text {cutHe+ }}$ ) can be obtained for a given group of $\eta_{s}$. Assuming that the boundary where the polarization reversal takes place (the blue solid curve) represents the trace of $f_{\text {crHe+ }}$, one can obtain $\eta_{\mathrm{H}^{+}} \sim 83 \%$ and $\eta_{\mathrm{He}^{+}} \sim 17 \%$. Substituting the obtained $\eta_{s}$ and the observed $\psi$ (Figure 2b) into Equations (2) and (7), one can obtain the theoretically estimated ellipticity $\epsilon_{B}^{*}$. As shown in Figure $2 \mathrm{~g}$, the polarization reversal boundary does exist for the $\epsilon_{B}^{*}$ spectra and generally matches the observed boundary. However, the waves observed before 03:20 UT can not be seen in the predicted spectra, suggesting that the cutoff frequencies are overestimated. Considering that the background plasma density decreases prominently with $L$ in the range where the wave exists (Figure $1 \mathrm{~d}$ ), one can assume that $\eta_{s}$ may also vary with $L$. Assuming that the cutoff frequencies lie just below the lowest frequencies of the observed intense waves, a group of $\eta_{s}$ can be obtained by Equation (8): $92 \% \mathrm{H}^{+}$and $8 \% \mathrm{He}^{+}$. As shown in Figure $2 \mathrm{~h}$, the predicted spectra contain the lower-frequency waves observed before 03:20 UT, but the predicted polarization reversal boundary does not match the observed boundary. In addition, a group of $\eta_{s}$ is provided in Figure $2 \mathrm{i}$ for comparison, where both the cutoff frequency and the polarization distribution deviate from the observations significantly. These results suggest that the $\mathrm{H}^{+}$abundance decreases from at least $92 \%$ at $L \sim 3.2$ to approximately $83 \%$ at $L \sim 3.5$ where the polarization reversal takes place. Furthermore, these results verify that the observed waves should belong to the unguided mode, and the polarization reversal boundary is indeed the time series of $f_{\mathrm{crHe}}+$

Two possible scenarios can explain the above phenomena. First, the source is in Region A, and the waves in Region B and Region C are the leaked waves from the source, as they are not guided by the ambient magnetic field. Second, the waves in Region A are parallelly excited while the waves in Region B and Region C are obliquely excited. 


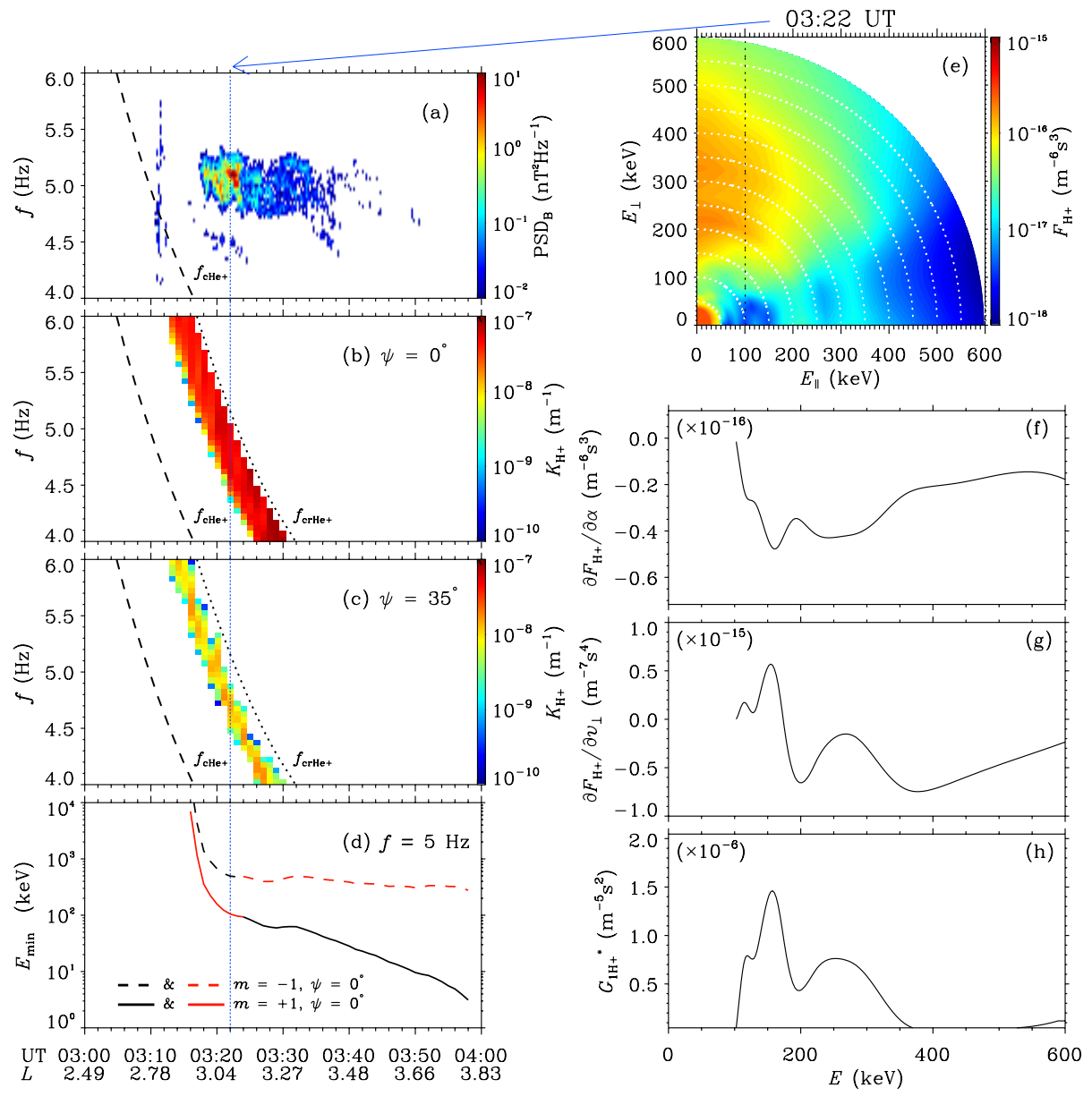

Figure 3: Wave growth analyses. (a) The observed magnetic power spectral density $\mathrm{PSD}_{B}$. (b-c) Wave convective growth rates $K_{\mathrm{H}+}$ contributed by $\mathrm{H}^{+}$at different wave normal angles. The dotted curves trace the $\mathrm{He}^{+}$crossover frequencies while the dashed curves trace the $\mathrm{He}^{+}$gyrofrequencies. (d) Minimum $\mathrm{H}^{+}$resonant energy $E_{\min }$ at a wave frequency of $f=5 \mathrm{~Hz}$ for different resonant orders. The red curves mark the resonance for the current unguided mode. (e) Distribution of the observed $\mathrm{H}^{+}$phase space density $F_{\mathrm{H}+}$ in the $E_{\perp}-E_{\|}$space at the time point 03:22 UT. The dotted line represents the resonant surface for the fundamental resonance at the wave normal angle $\psi=0^{\circ}$. (f-h) Energy dependence of $\partial F_{\mathrm{H}+} / \partial \alpha, \partial F_{\mathrm{H}+} / \partial v_{\perp}$ and $G_{1 \mathrm{H}+}^{*}$ along the resonant surface as shown in (e). Here $\alpha$ is the pitch angle, $v_{\perp}$ is the perpendicular velocity of $\mathrm{H}^{+}$, and $G_{1 \mathrm{H}+}^{*}$ is the integrating factor in the growth rate expression. 

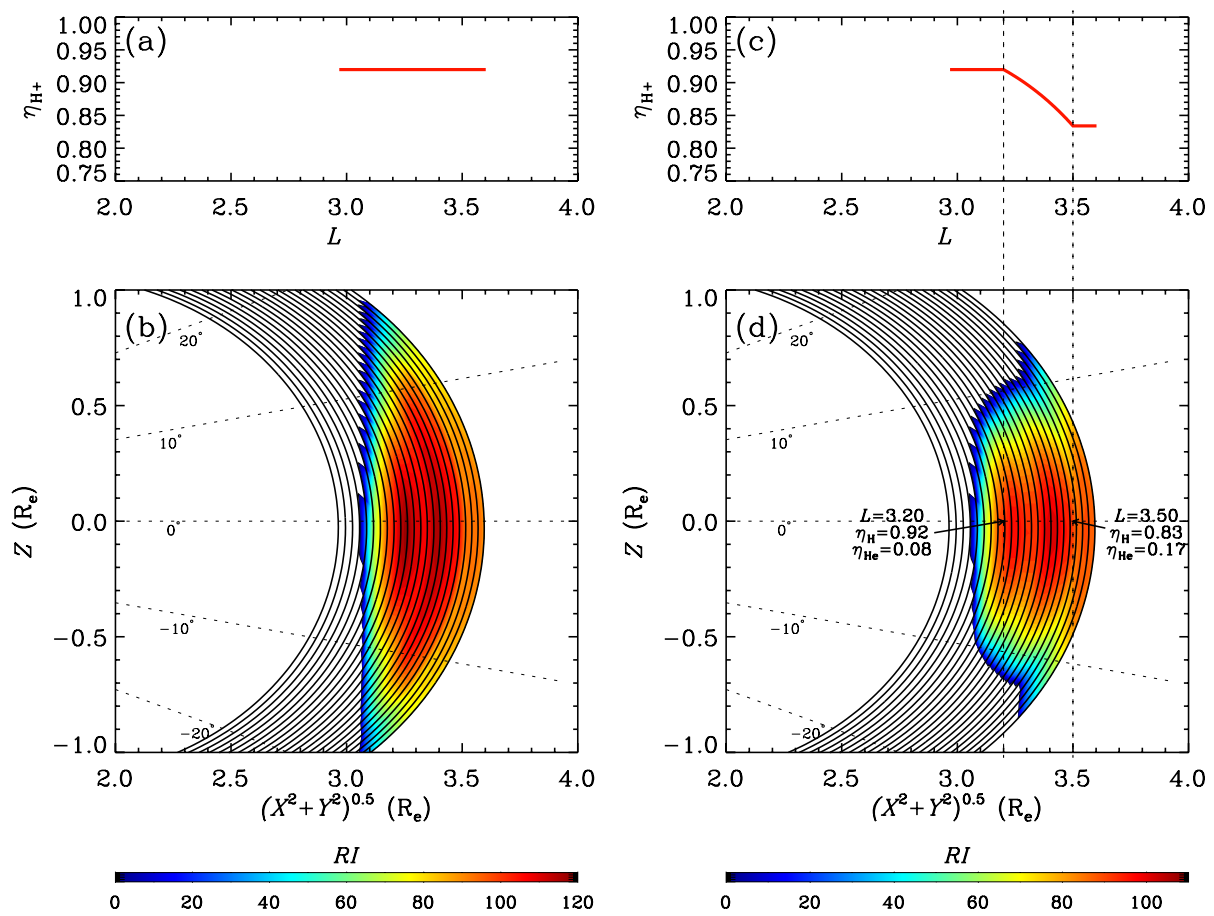

Figure 4: The distribution of the refractive index RI in a plasma environment with the ion abundance ratio $\eta_{\mathrm{H}+}$ : $(\mathrm{a}, \mathrm{b})$ distributed uniformly and $(\mathrm{c}, \mathrm{d})$ varied with the $L$-shell. The coordinate system is established in the Earth's dipole field: the horizontal axis represents the radial distance to the dipole axis, while the $Z$ axis is along the dipole axis. Solid black curves trace the background magnetic field estimated with the help of the T89 model. 


\section{Wave Growth Mechanism}

To reveal the generation mechanism of the observed unguided mode waves, we first present the results of the linear convective growth rate in Figure 3. Figure $3 \mathrm{~b}$ and Figure $3 \mathrm{c}$ show the convective growth rate distributions under the fixed $\eta_{s}: \eta_{\mathrm{H}^{+}}=92 \%$ and $\eta_{\mathrm{He}^{+}}=8 \%$. Positive growth rates appear below the local $f_{\mathrm{crHe}+}$, covering the ranges of the observed intense L-mode waves in the inner $L$-shell (Figure 3a). No wave growth can be obtained above $f_{\text {crHe+ }}$, due to the much higher resonant energy between $\mathrm{H}^{+}$and R-mode (Figure $3 \mathrm{~d}$ ). This result eliminates the possibility that the observed oblique R-mode waves in the outer $L$-shell are excited locally. However, the highest magnitude of the convective growth rate $K_{\mathrm{H}+}$ is $\sim 10^{-7} \mathrm{~m}^{-1}$ for the parallel waves, therefore, he path-integrated wave gain should be a factor of at least $\sim 10^{3}$ (7 e-foldings), to satisfy the growth of the observed intense waves from the background noise level (according to Figure 3a). This corresponds to an integrated traveling distance in the source adding up to $\sim 11 R_{\mathrm{E}}$.

Before the further explanation of wave growth, it is instructive to examine the exact potential source of the waves. Figure 3e displays the distribution of phase space density of proton $F_{\mathrm{H}+}$ and the resonant surface at 03:22 UT. As the minimum resonant energy is approximately $100 \mathrm{keV}$ for the fundamental resonance of the parallel left-hand waves, the resonant surface avoids the hot $\mathrm{H}^{+}$rings with lower energies, but crosses the ring with the energy range of $\sim$ $200-450 \mathrm{keV}$. The parameters crucial for the growth rate are presented in Figure 3f-h. The minimum of $\partial F_{\mathrm{H}+} / \partial \alpha$ and the maximum of $\partial F_{\mathrm{H}+} / \partial v_{\perp}$ are both obtained at $E_{k} \sim 180 \mathrm{keV}$, where the ring emerges (Figure $3 \mathrm{e}$ ). The final value of $G_{1 \mathrm{H}+}^{*}$ is positive from $100 \mathrm{keV}$ to $350 \mathrm{keV}$ and peaks at $E_{k} \sim 180 \mathrm{keV}$, indicating that these hot $\mathrm{H}^{+}$with several hundreds of $\mathrm{keV}$ contribute to the potential excitation of the most intense waves in Region A.

The waves within the inner $L$-shell are intense and predominated by fieldaligned wave vectors and Poynting fluxes but become weaker and more oblique outside. Moreover, the waves observed in all these regions have almost a constant frequency range. This evidence strongly implies that the waves are generated inside and propagated outward. The growth rate results further suggest this assumption by eliminating the possibility of oblique excitation for the outer right-hand waves. However, the most challenging problem is that the convective growth rate is too small for the observed strong waves generated in any potential source region with a limited spatial range. According to the previous analysis, an integrated traveling distance of $\sim 11 R_{\mathrm{E}}$ in the source region is needed for the full growth of the waves. It is reasonable to associate the convective growth process with some kind of wave trapping mechanism. As the hot ions are trapped in the narrow range of the $L$-shell, the azimuthal propagation of the waves may provide the required integrated distance in one-half of the drift circle. However, this scenario contradicts the observed parallel component of the Poynting fluxes. Considering that the intense quasi-parallel waves are just above the local cutoff frequency $f_{\text {cutHe+ }}$, where the unguided mode waves should be reflected. To investigate the possibility of wave trapping, the dis- 
tributions of refractive indexes RI are calculated in the simplified background plasma environment. The magnetic fields are estimated using the T89 model, and the values along each filed line are calibrated by multiplying the factor $B_{\text {obs }} / B_{\text {model }}$ (ratio of the observed and model estimated value at the intersection point between satellite orbit and field line). The density distribution is calculated by $\rho(M L A T)=\rho_{e q} / \cos (M L A T)$ with the help of the observations. As demonstrated in Figure $4 \mathrm{a}-\mathrm{b}$, in a plasma environment with constant $\eta_{s}$, the reflection interface where the refractive indexes RI decrease to zero is approximately perpendicular to the equatorial plane, because the value of $f_{\mathrm{cutHe}+} / f_{\mathrm{cH}+}$ remains the same while the value of $f_{\text {cutHe+ }}$ decreases with radial distance but increases with latitude due to the variation of the magnetic field. In the present case, the relative frequency $f_{\mathrm{cutHe}+} / f_{\mathrm{cH}+}$ should increase with $\eta_{\mathrm{He}+}$ (or decrease of $\eta_{\mathrm{H}+}$, as shown in Figure 4c) at a higher $L$-shell, thus offsetting the effect of the magnetic field. The strong reflection interface formed by the locations of $f_{\text {cutHe+ }}$ (where $\mathrm{RI}=0$ ) is therefore approximately parallel to the equatorial plane, as shown in Figure 4d. The conjugate reflection interfaces in both sides of the equatorial plane may produce a trapping zone, within which some of the unguided mode waves will be trapped and experience the significant amplification to the observed intensity. In addition, as shown in Figure $2 \mathrm{~b}$, the plasma number density experiences a sharp change at approximately $L=3.48$, which may result in a dramatic variation in $\eta_{s}$ with $L$, and thus form a quasi-parallel or even negatively inclined reflection interface at the radial distance $\sim 3.2-3.3$ $R_{\mathrm{E}}$. This is consistent with the strongest waves observed at $L \sim 3.2-3.3$ near the equator. Moreover, both the L-mode waves and the R-mode waves in Region B possess almost the same magnitude of wave power (Figure 2), suggesting that they are the leaked waves from the inner trapping region. Following such a scenario, one can predict that the waves observed away from the trapping region should propagate in the direction pointing from the trapping region to the observed position, which can explain the quasi-perpendicular waves observed in Region C.

\section{Discussion}

During the period from September 2013 to May 2018, another obvious event of unguided mode waves with a distinguishable crossover frequency was recorded by Probe A on 30 November 2015, when the satellite was outside the plasmasphere (please see Supplementary Fig.4). A similar trend of the spatial variation of ion abundance ratios can be obtained based on plasma diagnostics (Supplementary Fig.5), indicating the potential existence of wave trapping. The intense L-mode waves are observed along with the enhancement of hot $\mathrm{H}^{+}$with the energies of hundreds of $\mathrm{keV}$, while the positive convective growth rates are obtained (Supplementary Fig.6). The magnitude of largest growth rates is approximately several times of $10^{-7} \mathrm{~m}^{-1}$, which is inadequate for the waves to be amplified to the observed magnitude (more than 7 e-foldings) in an integrated distance less than $1 \mathrm{R}_{\mathrm{E}}$. As the waves are observed near the equatorial plane, 
one can thus predict that the wave trapping mechanism may have played an important role in the amplification of the observed waves.

The event in the previous section was recorded by Probe A. In fact, Probe B also recorded a wave event in the same area $\sim 2$ hours ago. As shown in Supplementary Fig.4, the intense $\mathrm{H}^{+}$band waves were observed at $L \sim 3.16, M L T \sim$ 8.5 and $M L A T \sim-1.2$, which were within exactly the same area of Probe-Aevent. Comparing Figure 1 and Supplementary Fig.7, the background plasma conditions are also similar for these two probes during their respective passes, i.e., waves were observed just outside the plasmasphere, with the enhancement of hot protons of hundreds of $\mathrm{keV}$. In the event of Probe B, a weak 'tail' toward the higher $L$-shell and larger value of $f / f_{\mathrm{cH}}+$ can also be observed. This 'tail' can be explained by the outward propagation of the unguided mode waves. In addition, the intense waves are separated by an area where the signals are weak (Supplementary Fig.7e). In the meanwhile, the spatial fluctuation of density is obvious (Supplementary Fig.7b), which may indicate the sharp variation of ion abundance ratios. In this event, however, no obvious boundary of R-mode and L-mode can be distinguished, as shown in Supplementary Fig.8. Therefore, we can not determine the mode of these waves based on the observations. However, as the plasma conditions are the same as that of Probe-A-event, and the weak 'tail' may indicate the propagation toward higher $L$-shell, it is reasonable to suppose that these waves are the unguided mode waves, being trapped and amplified with the same mechanism as the waves in Probe-A-event (Supplementary Fig.9 and Supplementary Fig.10 show the growth rates). If so, as these waves do not have an obvious right-hand polarization, they can not be distinguished from the guided mode waves, maybe they are common in the radiation belt and are intermixed with the guided mode waves in the previous studies. The exact measurements of ion abundance ratios are needed for further identification of these different modes.

From previous studies, the guided mode EMIC waves can scatter relativistic electrons via cyclotron resonance ${ }^{[48-52]}$ or bounce resonance ${ }^{[53]}$, and are finally damped by heating the $\sim 10 \mathrm{keV}$ ring current ions via harmonic cyclotron resonances ${ }^{[54]}$ or by heating the cold electrons up to a few hundred eV via Landau resonance ${ }^{[10,55]}$. In the present event, the wavenumber of the intense L-mode waves is approximately $1 \times 10^{-5} \mathrm{~m}^{-1}$ (Figure $4 \mathrm{f}$ ), and the non-relativistic electron gyrofrequency is approximately $24 \mathrm{kHz}$, therefore, the minimum resonant energy for the relativistic electrons is $\sim 10 \mathrm{MeV}$, corresponding to the highest energy of the relativistic electrons that have been detected [56]. In addition, as some waves could leak away from the source and propagate to the higher $L$-shell in an intermediate wave normal angle, if the background density does not decrease significantly, the parallel wavenumber should remain relatively unchanged while the electron gyrofrequency experiences a rapid decrease. In this case, one can predict a resonant scattering of the $\mathrm{MeV}$ electrons by the waves, in the core region of the radiation belt $(L \sim 4.0-5.0)$. In summary, the present study clearly demonstrates the existence of unguided mode EMIC waves in the radiation belt, and propose a trapping and amplification mechanism for explaining their origin. The results may have potential importance in the dynamics of 
the earth's radiation belt, and may provide inspiration for the investigations of the EMIC waves in the magnetosphere of other planets.

\section{Methods}

\section{Extraction of Wave Properties}

The magnetic field data from MAG, and the electric field data from EFW are used to analyze the wave properties. The $64 \mathrm{~Hz}$ magnetic field data are processed through a 1024-point fast Fourier transform (FFT, without detrending) to obtain the magnetic spectral matrix. Singular value decomposition (SVD) analysis [57] is performed on the magnetic spectral matrix to estimate the wave normal angle, azimuthal angle, and wave polarization ${ }^{[57]}$. The $32 \mathrm{~Hz}$ two-dimensional electric field data from EFW are used to estimate the vector electric field, and the spin axis-aligned component is estimated with the assumption that $\mathbf{E} \cdot \mathbf{B}=0$. Then, a 512-point FFT is carried out for both the electric field and the magnetic field (resampled to $32 \mathrm{~Hz}$ ) to obtain the cross-power spectra, and thus, we obtain the Poynting vector ${ }^{[58]}$.

\section{Plasma Diagnostics}

In the cold plasma, the following Stix parameters are helpful to investigate the dispersion relation [59]:

$$
L=1-\sum_{s} \frac{\omega_{\mathrm{p} s}^{2}}{\omega\left(\omega-\Omega_{s}\right)}, R=1-\sum_{s} \frac{\omega_{\mathrm{p} s}^{2}}{\omega\left(\omega+\Omega_{s}\right)}, P=1-\sum_{s}\left(\frac{\omega_{\mathrm{p} s}}{\omega}\right)^{2},
$$

here $\omega_{\mathrm{p} s}=\left(n_{s} q_{s}^{2} / m_{s} \varepsilon_{0}\right)^{\frac{1}{2}}$ and $\Omega_{s}=q_{s} B / m_{s}$ are respectively the plasma frequency and the gyrofrequency of a particle species s.

The crossover frequency $f_{\text {cr }}$ and the cutoff frequency $f_{\text {cut }}$ can connect the observations to the dispersion relation. $f_{\text {cr }}$ is the frequency where the L-mode and R-mode phase velocities are equal ${ }^{[60]}$ and can be obtained by setting the Stix parameter L equal to R:

$$
\sum_{s} \frac{\omega_{\mathrm{p} s}^{2}}{\omega_{\mathrm{cr}}\left(\omega_{\mathrm{cr}}-\Omega_{s}\right)}=\sum_{s} \frac{\omega_{\mathrm{p} s}^{2}}{\omega_{\mathrm{cr}}\left(\omega_{\mathrm{cr}}+\Omega_{s}\right)}
$$

Considering the charge neutrality condition, the determination of full ion abundance ratios requires the values of at least two characteristic frequencies (except gyrofrequencies) to be known in $\mathrm{H}^{+}, \mathrm{He}^{+}$and $\mathrm{O}^{+}$plasma. However, if the $\mathrm{O}^{+}$abundance $\eta_{\mathrm{O}+}$ is much lower than the $\mathrm{H}^{+}$abundance $\eta_{\mathrm{H}+}$ and if the focused mode belongs to the $\mathrm{H}^{+}$band which has a much larger frequency than the oxygen gyrofrequency $\Omega_{\mathrm{O}+}$, then we have the following relations:

$$
-\frac{\omega_{\mathrm{pH}+}^{2}}{\omega\left(\omega-\Omega_{\mathrm{H}+}\right)} \gg \frac{\omega_{\mathrm{pO}+}^{2}}{\omega\left(\omega-\Omega_{\mathrm{O}+}\right)} \text {, when } \Omega_{\mathrm{He}+}<\omega<\Omega_{\mathrm{H}+}
$$




$$
\frac{\omega_{\mathrm{pHe}+}^{2}}{\omega\left(\omega-\Omega_{\mathrm{He}+}\right)} \gg \frac{\omega_{\mathrm{pO}+}^{2}}{\omega\left(\omega-\Omega_{\mathrm{O}+}\right)} \text {, when } \omega \rightarrow \Omega_{\mathrm{He}+}
$$

313

$$
\frac{\omega_{\mathrm{pH}+}^{2}}{\omega\left(\omega+\Omega_{\mathrm{H}+}\right)} \gg \frac{\omega_{\mathrm{pO}+}^{2}}{\omega\left(\omega+\Omega_{\mathrm{O}+}\right)} \text {, when } \Omega_{\mathrm{He}+}<\omega<\Omega_{\mathrm{H}+}
$$

314

$$
\frac{\omega_{\mathrm{pH}+}^{2}}{\omega^{2}} \gg \frac{\omega_{\mathrm{pO}+}^{2}}{\omega^{2}} \text {, when } \Omega_{\mathrm{He}+}<\omega<\Omega_{\mathrm{H}+} .
$$

Therefore, if the three-ion $\left(\mathrm{H}^{+}, \mathrm{He}^{+}\right.$and $\left.\mathrm{O}^{+}\right)$plasma are approximated as twoion $\left(\mathrm{H}^{+}\right.$and $\left.\mathrm{He}^{+}\right)$plasma, i.e., the terms with oxygen plasma frequency $\omega_{\mathrm{pO}+}$ are dropped in Equation (1), the Stix parameters will have only a negligible change (example in Supplementary Fig.2). Under such an approximation, a group of ion abundance ratios $\left(\eta_{\mathrm{H}+}\right.$ and $\left.\eta_{\mathrm{He}+}\right)$ can be obtained by substituting the observed value of $\omega_{\mathrm{crHe}+} / \Omega_{\mathrm{H}+}$ into Equation (2). Consequently, the approximated dispersion relations for the modes in $\mathrm{H}^{+}$band can be found.

As the wave magnetic field vector is always perpendicular to the wave vector (i.e., $\mathbf{B} \cdot \mathbf{k}=0$ ), it is reasonable to define the polarization in terms of the wave magnetic field, which is perpendicular to $\mathbf{k}$. As the sense of wave field rotation about the ambient magnetic field $\mathbf{B}_{\mathbf{0}}$ remains the same for a certain polarization mode, it is convenient to define the handedness in terms of $\mathbf{B}_{\mathbf{0}}$. Based on these considerations, the polarization state can be represented by the ellipticity $\epsilon_{B}$, where negative values represent left-hand polarization (see Supplementary Figure 1). $\epsilon_{B}$ changes sign upon crossing $f_{\mathrm{cr}}$. For the observed polarization reversal, a validation of whether it represents the crossover frequency $f_{\text {cr }}$ can be performed by comparing the distribution of the observed ellipticity $\epsilon_{B}$ with the theoretically predicted ellipticity $\epsilon_{B}^{*}$. In the coordinate system with the z-axis along $\mathbf{B}_{\mathbf{0}}$ and the $\mathrm{y}$-axis perpendicular to the $\mathbf{B}_{\mathbf{0}}-\mathbf{k}$ plane, the ratio of the wave magnetic field can be expressed as ${ }^{[61,62]}$ :

$$
\frac{B_{x}}{B_{y}}=-\frac{(R-L)\left(P-n^{2} \sin ^{2} \psi\right)}{P\left(R+L-2 n^{2}\right)},
$$

where $\psi$ is the wave normal angle. The field amplitude can be transformed into the coordinate system with the $\mathrm{z}$-axis along $\mathbf{k}$ :

$$
B_{x}^{\prime}=\frac{B_{x}}{\cos \psi}, \quad B_{y}^{\prime}=B_{y},
$$

the theoretically predicted ellipticity $\epsilon_{B}^{*}$ is then obtained considering that $B_{x}^{\prime}$ and $B_{y}^{\prime}$ are axes of the polarization ellipse.

In summary, the ion abundance ratios are estimated as follows: first, estimate the potential crossover frequency from the observed polarization of $\mathrm{H}^{+}$ band waves; then, estimate the $\mathrm{H}^{+}$and $\mathrm{He}^{+}$abundance ratios by Equation 
(1); finally, compare the observed ellipticity $\epsilon_{B}$ with the calculated theoretical ellipticity $\epsilon_{B}^{*}$ for verification.

The cutoff frequency $f_{\text {cut }}$ is the frequency where the phase velocity equals to zero [smith1964propagation], and can be obtained by setting the Stix parameter L equal to zero:

$$
1-\sum_{s} \frac{\omega_{\mathrm{p} s}^{2}}{\omega_{\text {cut }}\left(\omega_{\text {cut }}-\Omega_{s}\right)}=0
$$

Theoretically, the vanishing of waves below the lowest frequency of the observed wave power spectrum (denoted as $f_{\min }$ ) may be simply a result of a decrease of wave growth, which is due to the hot ion distribution and resonant conditions rather than the effect of the cutoff frequency. Therefore, the lower limit of the $\mathrm{H}^{+}$abundance ratio can be estimated by inserting the relation $f_{\text {min }} \geq f_{\text {cut }}$ into Equation (9).

\section{The Convective Growth Rate for Unguided Mode around $\mathrm{H}^{+}$Gyrofrequency}

For a given ion phase space density distribution $F\left(v_{\|}, v_{\perp}\right)$, the growth rate depends on the integration of the terms containing $\partial F\left(v_{\|}, v_{\perp}\right) / \partial v_{\perp}$ and $\partial F\left(v_{\|}, v_{\perp}\right) / \partial v_{\|}$ along the resonant surface $v_{\|}=\left(\omega-m \Omega_{s}\right) / k_{\|}$. Based on Kennel et al. ${ }^{[63]}$, here, we derive a simplified form of the convective growth rate for the unguided mode near $\mathrm{H}^{+}$gyrofrequency to better demontrate how the $F\left(v_{\|}, v_{\perp}\right)$ distribution leads to wave growth. In the environment of the radiation belt, the Stix parameter $P$ dominates $L, R$ and refractive index $R_{I}$. One can thus simplify the imaginary part of the first order of the dispersion function:

$$
\begin{aligned}
\operatorname{Im} D^{(1)}(\omega, \mathbf{k})= & -2 \pi \sum_{s} \frac{\omega_{\mathrm{p} s}^{2}}{\omega\left|k_{\|}\right|} \int_{0}^{\infty} v_{\perp} d v_{\perp} \int_{-\infty}^{+\infty} d v_{\|} \sum_{m} \delta\left(v_{\|}-\frac{\omega-m \Omega_{s}}{k_{\|}}\right) \\
& \cdot 2 P v_{\perp}\left[R+L-2 R_{I}^{2}\right] G_{1 s}^{*}
\end{aligned}
$$

where

$$
\begin{aligned}
G_{1 s}^{*} & \approx G_{1 s} \frac{1}{2\left[R+L-2 R_{I}^{2}\right]}\left[2\left(L-R_{I}^{2}\right) J_{m+1}^{2}+2\left(R-n^{2}\right) J_{m-1}^{2}+R_{I}^{2} \sin ^{2} \psi\left(J_{m+1}-J_{m-1}\right)^{2}\right] \\
& =\left(\frac{\partial \bar{F}_{s}}{\partial v_{\perp}}-\frac{k_{\|}}{\omega} \frac{\partial \bar{F}_{s}}{\partial \alpha}\right) \cdot\left[\frac{L-R_{I}^{2}}{R+L-2 R_{I}^{2}} J_{m+1}+\frac{R-R_{I}^{2}}{R+L-2 R_{I}^{2}} J_{m-1}\right]^{2}
\end{aligned}
$$

Following ${ }^{[64]}$, express the magnitude of convective growth rate $\mathbf{k}_{\mathbf{i}}$ as

$$
k_{i}=-\frac{\operatorname{Im} D^{(1)}(\omega, \mathbf{k})}{\widehat{\mathbf{k}} \cdot \partial D^{(0)} / \partial \mathbf{k}},
$$

here

$$
\frac{\partial D^{(0)}}{\partial k}=\frac{8 P}{k}\left[\cos ^{2} \psi R_{I}^{4}-R L\right]
$$


Considering that the group velocity points in nearly the same direction as the wave vector for the unguided mode, one can finally express the component of $\mathbf{k}_{\mathbf{i}}$ in the group velocity direction as:

$$
\begin{aligned}
K_{i} & =-\hat{\mathbf{k}} \cdot \hat{\mathbf{v}}_{\mathbf{g}} k_{i} \\
& \approx-k_{i} \\
& \approx \frac{1}{2} \pi^{2} \sum_{s} \frac{k \omega_{\mathrm{p} s}^{2}}{\left|k_{\|}\right| \omega+L-2 R_{I}^{2}} \frac{R+\cos ^{2} \psi R_{I}^{4}}{R L} \int_{0}^{\infty} v_{\perp}^{2} G_{1 s}^{*} d v_{\perp} .
\end{aligned}
$$

It can be proven that the product of the factors outside the integral is greater than zero, therefore, the values of $G_{1 s}^{*}$ represent the contribution to wave growth by hot ions with a given energy range.

The derivatives of the phase space density $\left(F=j / p^{2}\right)$ with respect to the energy $E$ and pitch angle $\alpha$ for each ion species can be obtained based on observation. As the major ions $\mathrm{H}^{+}$dominate the wave growth, here the phase space density $F$ is estimated approximately from the $\mathrm{H}^{+}$flux collected by HOPE and RBSPICE. HOPE measures the $\mathrm{H}^{+}$flux $\left(j_{\mathrm{H}+}\right)$ with the energy from several $\mathrm{eV}$ to $\sim 50 \mathrm{keV}$, while the RBSPICE measures $j_{\mathrm{H}+}$ from $\sim 50 \mathrm{keV}$ to $\sim 600 \mathrm{keV}$. These two energy ranges cover the main structures of the ring current $\mathrm{H}^{+}$and are sufficient to evaluate the linear instability. The HOPE flux is multiplied by a factor of 3 so that the mismatch between the low-energy part of RBSPICE and the high-energy part of HOPE can be eliminated $[65,66]$. The distribution of $F(E, \alpha)$ is fitted along pitch angle $\alpha$ for each fixed energy channel, and the required derivatives are then approximated with the help of B-spline interpolation. The details regarding this technique follow ${ }^{[67]} . D^{(0)}$ and $D^{(1)}$ also depend on the background magnetic field, the plasma number density $n_{e}$ and the ion abundance ratios $\eta_{s}=n_{s} / n_{e}$ (where s denotes the particle species). The magnetic field is measured by MAG, the local plasma density is measured HFR, and the ion abundance ratios are determined via the plasma diagnostics.

\section{Data Availability}

The particle data are available from http://www.RBSP-ect.lanl.gov/ and http://rbspice.ftecs.com/Data.html. The electric field data are available from http://www.space.umn.edu/rbspefw-data/. The magnetic field data are available from http://emfisis.physics.uiowa.edu/Flight/. The magnetic field model can be obtained from http://geo.phys.spbu.ru/\%7etsyganenko/ modeling.html. The geomagnetic indices are available from https://omniweb. gsfc.nasa.gov/

\section{References}

[1] Yoshiya Kasahara et al. "Ion cyclotron emissions observed by the satellite Akebono in the vicinity of the magnetic equator". In: Radio Sci. 27.2 (Apr. 1992), pp. 347-362. DOI: 10.1029/91RS01872. 
[2] K. Keika et al. "Global characteristics of electromagnetic ion cyclotron waves: Occurrence rate and its storm dependence". In: J. Geophys. Res. (Space Physics) 118 (July 2013), pp. 4135-4150. DOI: 10.1002/jgra . 50385.

[3] N. P. Meredith et al. "Global morphology and spectral properties of EMIC waves derived from CRRES observations". In: J. Geophys. Res. (Space Physics) 119 (July 2014), pp. 5328-5342. DOI: 10.1002/2014JA020064.

[4] Xiongdong $\mathrm{Yu}$ et al. "In situ observations of EMIC waves in $\mathrm{O}^{+}$band by the Van Allen Probe A". In: Geophys. Res. Lett. 42.5 (Mar. 2015), pp. 1312-1317. DOI: 10.1002/2015GL063250.

[5] A. A. Saikin et al. "The occurrence and wave properties of $\mathrm{H}^{+}-\mathrm{He}^{+}-$ , and $\mathrm{O}^{+}$-band EMIC waves observed by the Van Allen Probes". In: $J$. Geophys. Res. (Space Physics) 120 (Sept. 2015), pp. 7477-7492. DOI: 10. 1002/2015JA021358.

[6] D. Wang et al. "Statistical characteristics of EMIC waves: Van Allen Probe observations". In: J. Geophys. Res. (Space Physics) 120 (June 2015), pp. 4400-4408. DOI: $10.1002 / 2015$ JA021089.

[7] X. Y. Wang et al. "The occurrence and wave properties of EMIC waves observed by the Magnetospheric Multiscale (MMS) mission". In: J. Geophys. Res. (Space Physics) 122.8 (Aug. 2017), pp. 8228-8240. DOI: 10 . 1002/2017JA024237.

[8] Huayue Chen et al. "Analyzing EMIC Waves in the Inner Magnetosphere Using Long-Term Van Allen Probes Observations". In: J. Geophys. Res. (Space Physics) 124.9 (Sept. 2019), pp. 7402-7412. DOI: 10.1029/2019JA026965.

[9] S. S. Tetrick et al. "Location of intense electromagnetic ion cyclotron (EMIC) wave events relative to the plasmapause: Van Allen Probes observations". In: J. Geophys. Res. (Space Physics) 122 (Apr. 2017), pp. 40644088. DOI: $10.1002 / 2016$ JA023392.

[10] R. M. Thorne et al. "Interaction of EMIC Waves With Thermal Plasma and Radiation Belt Particles". In: Magnetospheric ULF Waves: Synthesis and New Directions. Ed. by K. Takahashi et al. Vol. 169. Washington DC American Geophysical Union Geophysical Monograph Series. 2006, p. 213. DOI: $10.1029 / 169$ GM14.

[11] Y. Miyoshi et al. "Precipitation of radiation belt electrons by EMIC waves, observed from ground and space". In: Geophys. Res. Lett. 35, L23101 (Dec. 2008), p. 23101. DOI: 10.1029/2008GL035727.

[12] Z. Wang et al. "Statistical characteristics of EMIC wave-driven relativistic electron precipitation with observations of POES satellites: Revisit". In: J. Geophys. Res. (Space Physics) 119 (July 2014), pp. 5509-5519. DOI: 10.1002/2014JA020082. 
[13] M. E. Usanova et al. "Effect of EMIC waves on relativistic and ultrarelativistic electron populations: Ground-based and Van Allen Probes observations". In: Geophys. Res. Lett. 41 (Mar. 2014), pp. 1375-1381. DOI: 10.1002/2013GL059024.

[14] L. W. Blum et al. "Observations of coincident EMIC wave activity and duskside energetic electron precipitation on 18-19 January 2013". In: Geophys. Res. Lett. 42.14 (July 2015), pp. 5727-5735. DOI: 10.1002/2015GL065245.

[15] C. J. Rodger et al. "High-resolution in situ observations of electron precipitationcausing EMIC waves". In: Geophys. Res. Lett. 42 (Nov. 2015), pp. 96339641. DOI: $10.1002 / 2015$ GL066581.

[16] X. J. Zhang et al. "Physical mechanism causing rapid changes in ultrarelativistic electron pitch angle distributions right after a shock arrival: Evaluation of an electron dropout event". In: J. Geophys. Res. (Space Physics) 121.9 (Sept. 2016), pp. 8300-8316. DOI: 10.1002/2016JA022517.

[17] Z. Su et al. "Rapid Loss of Radiation Belt Relativistic Electrons by EMIC Waves". In: J. Geophys. Res. (Space Physics) 122 (Oct. 2017), pp. 98809897. DOI: $10.1002 / 2017$ JA024169.

[18] L. Bingley et al. "The Evolution of a Pitch-Angle "Bite-Out" Scattering Signature Caused by EMIC Wave Activity: A Case Study". In: J. Geophys. Res. (Space Physics) 124.7 (July 2019), pp. 5042-5055. DOI: 10.1029/ 2018JA026292.

[19] L. W. Blum et al. "Persistent EMIC Wave Activity Across the Nightside Inner Magnetosphere". In: Geophys. Res. Lett. 47.6, e87009 (Mar. 2020), e87009. DOI: $10.1029 / 2020$ GL087009.

[20] Hui Zhu et al. "Direct Evidence of the Pitch Angle Scattering of Relativistic Electrons Induced by EMIC Waves". In: Geophys. Res. Lett. 47.4, e85637 (Feb. 2020), e85637. DOI: 10.1029/2019GL085637.

[21] J. L. Rauch and A. Roux. "Ray tracing of ULF waves in a multicomponent magnetospheric plasma - Consequences for the generation mechanism of ion cyclotron waves". In: J. Geophys. Res. 87 (Oct. 1982), pp. 8191-8198. DOI: 10.1029/JA087iA10p08191.

[22] R. B. Horne and R. M. Thorne. "On the preferred source location for the convective amplification of ion cyclotron waves". In: J. Geophys. Res. 98 (June 1993), pp. 9233-9247. DOI: 10.1029/92JA02972.

[23] John M. Cornwall. "Cyclotron Instabilities and Electromagnetic Emission in the Ultra Low Frequency and Very Low Frequency Ranges". In: $J$. Geophys. Res. (Space Physics) 70.1 (Jan. 1965), pp. 61-69. DOI: 10.1029/ JZ070i001p00061.

[24] C. F. Kennel and H. E. Petschek. "Limit on Stably Trapped Particle Fluxes". In: J. Geophys. Res. 71 (Jan. 1966), p. 1.

[25] B. J. Anderson and D. C. Hamilton. "Electromagnetic ion cyclotron waves stimulated by modest magnetospheric compressions". In: J. Geophys. Res. (Space Physics) 98 (July 1993), p. 11. DOI: 10.1029/93JA00605. 
[26] L. Chen et al. "Global simulation of EMIC wave excitation during the 21 April 2001 storm from coupled RCM-RAM-HOTRAY modeling". In: $J$. Geophys. Res. 115, A07209 (July 2010), p. 7209. DOI: 10.1029/2009JA015075.

[27] Manish Mithaiwala et al. "Convective amplification of electromagnetic ion cyclotron waves from ring-distribution protons in the inner magnetosphere". In: J. Geophys. Res. (Space Physics) 118.12 (Dec. 2013), pp. 7538-7544. DOI: 10.1002/2013JA019134. arXiv:1306.2215 [physics.space-ph].

[28] X. Yu et al. "Excitation of oblique $\mathrm{O}^{+}$band EMIC waves in the inner magnetosphere driven by hot $\mathrm{H}^{+}$with ring velocity distributions". In: $J$. Geophys. Res. (Space Physics) 121 (Nov. 2016), p. 11. DOI: 10.1002 / 2016JA023221.

[29] M. F. Thomsen et al. "Ring/Shell Ion Distributions at Geosynchronous Orbit". In: J. Geophys. Res. (Space Physics) 122.12 (Dec. 2017), pp. 12,05512,071. DOI: $10.1002 / 2017$ JA024612.

[30] B. Remya et al. "Ion Injection Triggered EMIC Waves in the Earth's Magnetosphere". In: J. Geophys. Res. (Space Physics) 123 (June 2018), pp. 4921-4938. DOI: $10.1029 / 2018$ JA025354.

[31] J. U. Kozyra et al. "Effects of energetic heavy ions on electromagnetic ion cyclotron wave generation in the plasmapause region". In: J. Geophys. Res. (Space Physics) 89.A4 (Apr. 1984), pp. 2217-2234. DOI: 10.1029/ JA089iA04p02217.

[32] S. Peter Gary. Theory of Space Plasma Microinstabilities. 1993.

[33] M. J. Engebretson et al. "Van Allen probes, NOAA, GOES, and ground observations of an intense EMIC wave event extending over $12 \mathrm{~h}$ in magnetic local time". In: J. Geophys. Res. (Space Physics) 120 (July 2015), pp. 5465-5488. DOI: 10.1002/2015JA021227.

[34] L. W. Blum et al. "EMIC wave scale size in the inner magnetosphere: Observations from the dual Van Allen Probes". In: Geophys. Res. Lett. 44 (Feb. 2017), pp. 1227-1233. DOI: 10.1002/2016GL072316.

[35] Si Liu et al. "Magnetospheric Multiscale Observation of Quasiperiodic EMIC Waves Associated With Enhanced Solar Wind Pressure". In: Geophys. Res. Lett. 46.13 (July 2019), pp. 7096-7104. DOI: 10.1029 / 2019 GL083421.

[36] L. Chen, R. M. Thorne, and R. B. Horne. "Simulation of EMIC wave excitation in a model magnetosphere including structured high-density plumes". In: J. Geophys. Res. (Space Physics) 114, A07221 (July 2009), A07221. DOI: 10.1029/2009JA014204.

[37] M. de Soria-Santacruz, M. Spasojevic, and L. Chen. "EMIC waves growth and guiding in the presence of cold plasma density irregularities". In: Geophys. Res. Lett. 40 (May 2013), pp. 1940-1944. DOI: 10.1002/grl. 50484 . 
[38] J.-C. Zhang et al. "Excitation of EMIC waves detected by the Van Allen Probes on 28 April 2013". In: Geophys. Res. Lett. 41 (June 2014), pp. 4101-4108. DOI: 10.1002/2014GL060621.

[39] R. C. Allen et al. "A statistical study of EMIC waves observed by Cluster: 2. Associated plasma conditions". In: J. Geophys. Res. (Space Physics) 121.7 (July 2016), pp. 6458-6479. DOI: 10.1002/2016JA022541.

[40] Zhigang Yuan et al. "Simultaneous trapping of EMIC and MS waves by background plasmas". In: J. Geophys. Res. (Space Physics) 0.ja (2019). DOI: $10.1029 / 2018 \mathrm{JA} 026149$.

[41] H. Zhao et al. "The evolution of ring current ion energy density and energy content during geomagnetic storms based on Van Allen Probes measurements". In: J. Geophys. Res. (Space Physics) 120.9 (Sept. 2015), pp. 74937511. DOI: 10.1002/2015JA021533.

[42] R. B. Horne and R. M. Thorne. "Convective instabilities of electromagnetic ion cyclotron waves in the outer magnetosphere". In: J. Geophys. Res. 99 (Sept. 1994), p. 17259. DOI: 10.1029/94JA01259.

[43] B. H. Mauk et al. "Science Objectives and Rationale for the Radiation Belt Storm Probes Mission". In: Space. Sci. Rev. 179 (Nov. 2013), pp. $3-$ 27. DOI: $10.1007 / \mathrm{s} 11214-012-9908-y$.

[44] D. G. Mitchell et al. "Radiation Belt Storm Probes Ion Composition Experiment (RBSPICE)". In: Space Sci. Rev. 179.1-4 (Nov. 2013), pp. 263308. DOI: $10.1007 / \mathrm{s} 11214-013-9965-\mathrm{x}$.

[45] H. O. Funsten et al. "Helium, Oxygen, Proton, and Electron (HOPE) Mass Spectrometer for the Radiation Belt Storm Probes Mission". In: Space. Sci. Rev. 179 (Nov. 2013), pp. 423-484. DOI: $10.1007 /$ s11214013-9968-7.

[46] W. S. Kurth et al. "Electron densities inferred from plasma wave spectra obtained by the Waves instrument on Van Allen Probes". In: J. Geophys. Res. (Space Physics) 120 (Feb. 2015), pp. 904-914. DOI: 10.1002/ 2014 JA020857.

[47] C. A. Kletzing et al. "The Electric and Magnetic Field Instrument Suite and Integrated Science (EMFISIS) on RBSP". In: Space. Sci. Rev. 179 (Nov. 2013), pp. 127-181. DOI: 10.1007/s11214-013-9993-6.

[48] B. Ni et al. "Resonant scattering of outer zone relativistic electrons by multiband EMIC waves and resultant electron loss time scales". In: $J$. Geophys. Res. (Space Physics) 120 (Sept. 2015), pp. 7357-7373. DOI: 10. 1002/2015JA021466.

[49] B. Wang et al. "Nonlinear Landau resonant scattering of near-equatorially mirroring radiation belt electrons by oblique EMIC waves". In: Geophys. Res. Lett. 43 (2016), pp. 3628-3636. DOI: 10.1002/2016GL068467. 
[50] G. Wang et al. "Nonlinear fundamental and harmonic cyclotron resonant scattering of radiation belt ultrarelativistic electrons by oblique monochromatic EMIC waves". In: J. Geophys. Res. (Space Physics) 122 (Feb. 2017), pp. 1928-1945. DOI: 10.1002/2016JA023451.

[51] Dae-Young Lee, Dae-Kyu Shin, and Cheong-Rim Choi. "Effects of Oblique Wave Normal Angle and Noncircular Polarization of Electromagnetic Ion Cyclotron Waves on the Pitch Angle Scattering of Relativistic Electrons". In: J. Geophys. Res. (Space Physics) 123.6 (June 2018), pp. 4556-4573. DOI: $10.1029 / 2018 \mathrm{JA} 025342$.

[52] Song $\mathrm{Fu}$ et al. "Interactions between $\mathrm{H}^{+}$band EMIC waves and radiation belt relativistic electrons: Comparisons of test particle simulations with quasi-linear calculations". In: Phys. Plasmas 26.3, 032901 (2019), p. 032901. DOI: $10.1063 / 1.5054809$.

[53] Xing Cao et al. "Bounce resonance scattering of radiation belt electrons by $\mathrm{H}^{+}$band EMIC waves". In: J. Geophys. Res. (Space Physics) 122.2 (2017), pp. 1702-1713. DOI: 10.1002/2016JA023607.

[54] X. Cao et al. "Sensitivity of EMIC Wave-Driven Scattering Loss of Ring Current Protons to Wave Normal Angle Distribution". In: Geophys. Res. Lett. 46 (Jan. 2019), pp. 590-598. DOI: 10.1029/2018GL081550.

[55] Z. Yuan et al. "Cold electron heating by EMIC waves in the plasmaspheric plume with observations of the Cluster satellite". In: Geophys. Res. Lett. 41 (Mar. 2014), pp. 1830-1837. DOI: 10.1002/2014GL059241.

[56] Ch. Katsavrias et al. "Highly Relativistic Electron Flux Enhancement During the Weak Geomagnetic Storm of April-May 2017". In: J. Geophys. Res. (Space Physics) 124.6 (June 2019), pp. 4402-4413. DOI: 10.1029/ 2019JA026743. arXiv:1902.00960 [physics.space-ph].

[57] O. Santolík, M. Parrot, and F. Lefeuvre. "Singular value decomposition methods for wave propagation analysis". In: Radio Sci. 38, 1010 (Feb. 2003), p. 1010. DOI: $10.1029 / 2000$ RS002523.

[58] O. Santolík et al. "Survey of Poynting flux of whistler mode chorus in the outer zone". In: J. Geophys. Res. (Space Physics) 115, A00F13 (July 2010), A00F13. DOI: 10.1029/2009JA014925.

[59] T. H. Stix. Waves in Plasmas. New York: American Institute of Physics, 1992.

[60] R. L. Smith and Neil Brice. "Propagation in Multicomponent Plasmas". In: J. Geophys. Res. 69.23 (Dec. 1964), pp. 5029-5040. DOI: 10.1029/ JZ069i023p05029.

[61] X. Tao and J. Bortnik. "Nonlinear interactions between relativistic radiation belt electrons and oblique whistler mode waves". In: Nonlinear Proc. Geoph. 17 (Oct. 2010), pp. 599-604. DOI: 10.5194/npg-17-599-2010. 
[62] J. Bortnik and R. M. Thorne. "Transit time scattering of energetic electrons due to equatorially confined magnetosonic waves". In: J. Geophys. Res. (Space Physics) 115, A07213 (July 2010), A07213. DOI: 10.1029/ 2010 JA015283.

[63] C. Kennel. "Low-Frequency Whistler Mode". In: Phys. Fluids 9 (Nov. 1966), pp. 2190-2202. DOI: 10.1063/1.1761588.

[64] L. Chen et al. "Global simulation of magnetosonic wave instability in the storm time magnetosphere". In: J. Geophys. Res. (Space Physics) 115, A11222 (Nov. 2010), A11222. DOI: 10.1029/2010JA015707.

[65] Kyungguk Min et al. "Second harmonic poloidal waves observed by Van Allen Probes in the dusk-midnight sector". In: J. Geophys. Res. (Space Physics) 122.3 (Mar. 2017), pp. 3013-3039. DOI: 10.1002/2016JA023770.

[66] L. M. Kistler et al. "The source of $\mathrm{O}^{+}$in the storm time ring current". In: J. Geophys. Res. (Space Physics) 121.6 (June 2016), pp. 5333-5349. DOI: 10.1002/2015JA022204.

[67] N. Liu et al. "Prompt Disappearance and Emergence of Radiation Belt Magnetosonic Waves Induced by Solar Wind Dynamic Pressure Variations". In: Geophys. Res. Lett. 45 (Jan. 2018), pp. 585-594. DOI: 10.1002/ 2017 GL076382. 


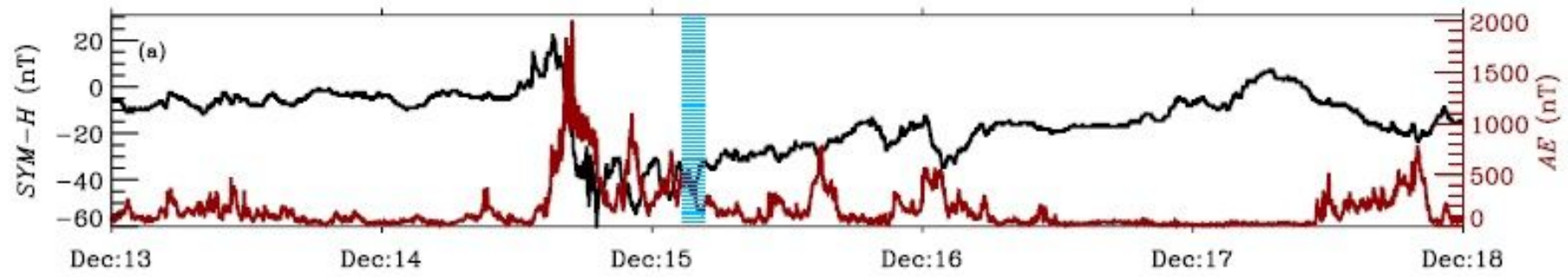

2015-12-15 Van Allen Probe-A

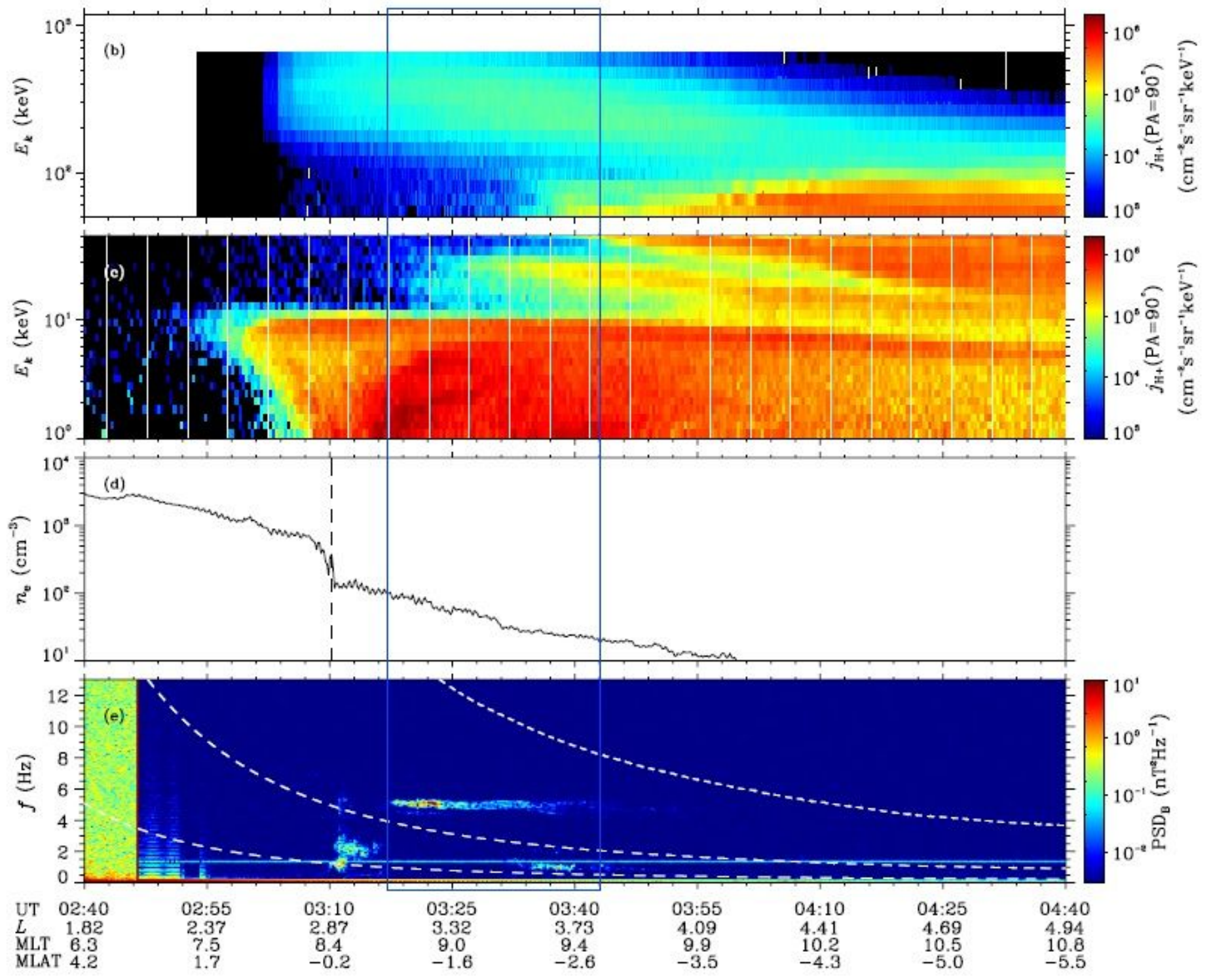

Figure 1

Overview of the background conditions of the wave event recorded by Van Allen Probe A on 15 December 2015 (see Manuscript file for full caption.) 


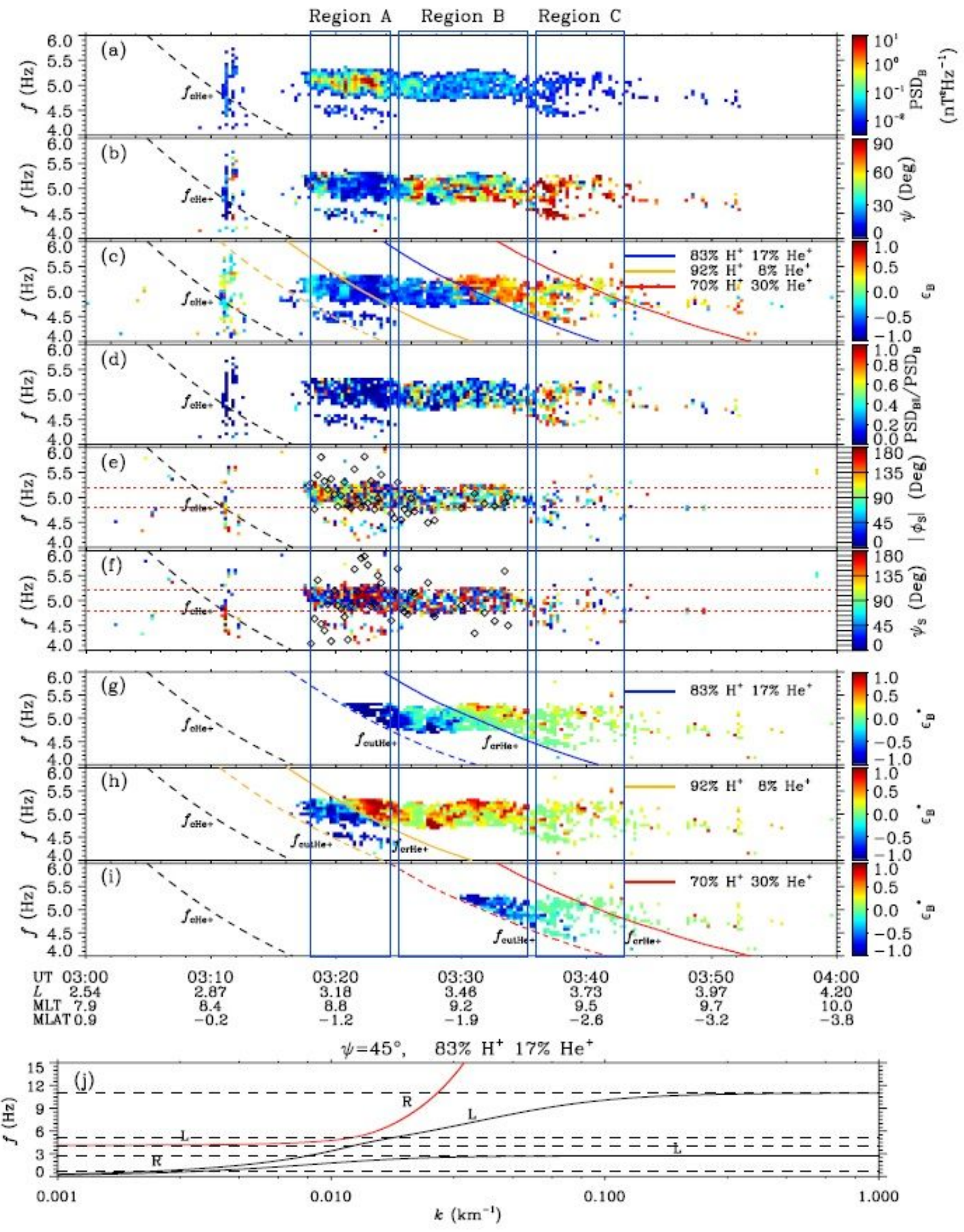

Figure 2

Analyses of wave properties and wave mode. (see Manuscript file for full caption.) 


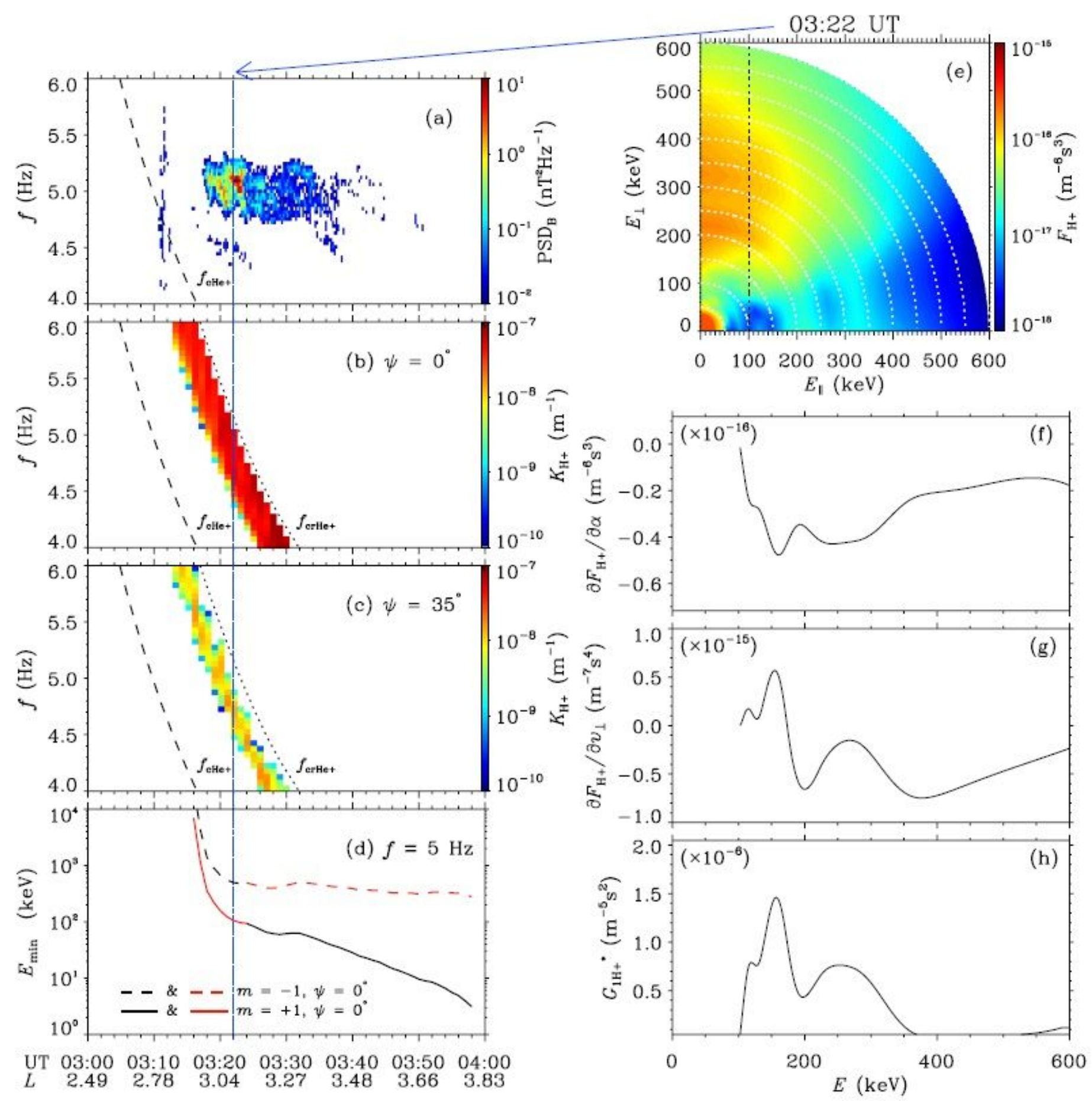

Figure 3

Wave growth analyses. (see Manuscript file for full caption.) 

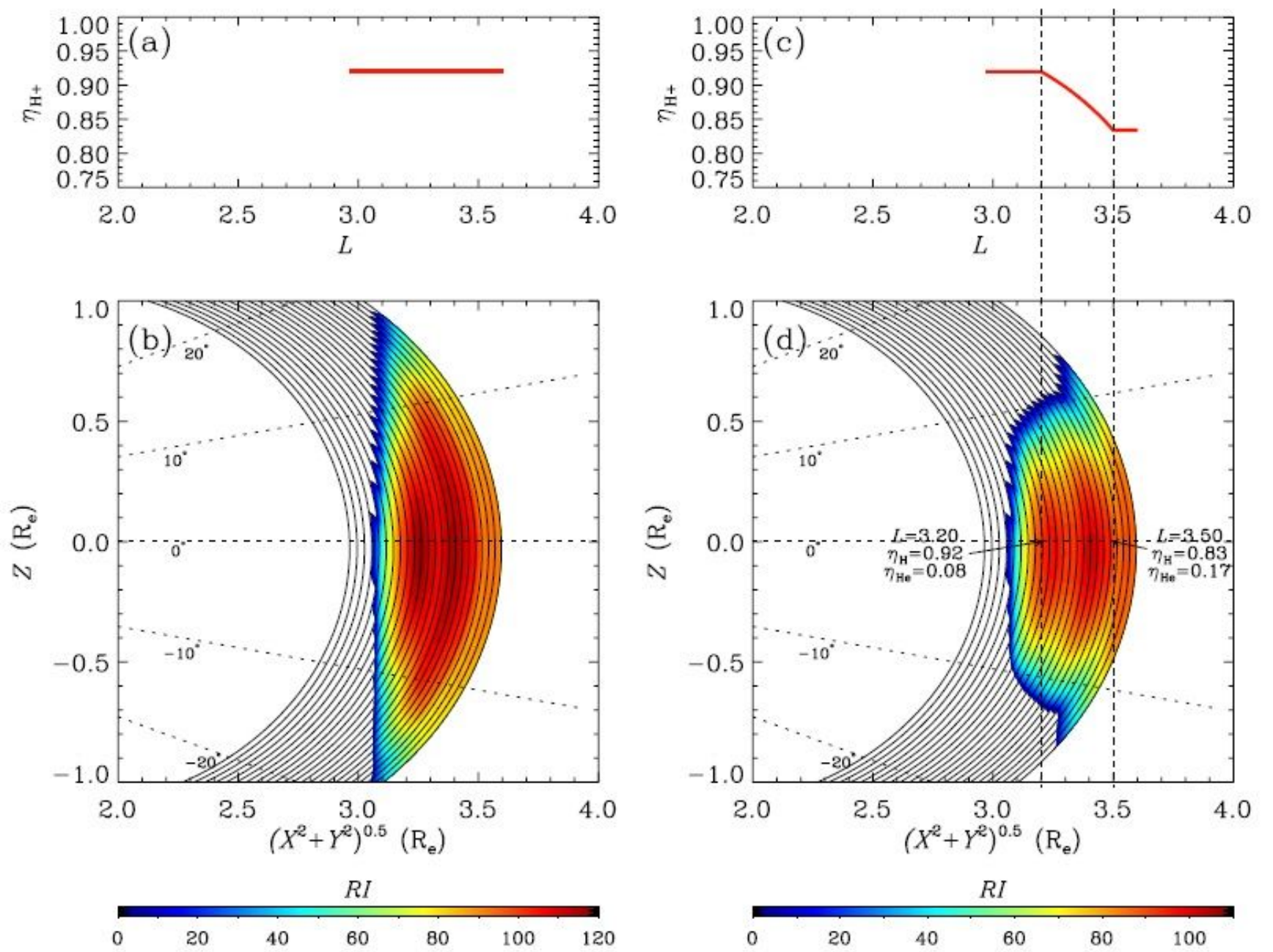

\section{Figure 4}

The distribution of the refractive index $\mathrm{Rl}$ in a plasma environment with the ion abundance ratio $\mathrm{nH}+$ (see Manuscript file for full caption.)

\section{Supplementary Files}

This is a list of supplementary files associated with this preprint. Click to download.

- supplementarydocument.pdf 